\title{
DEGREES OF IRREDUCIBLE MORPHISMS IN GENERALIZED STANDARD COHERENT ALMOST CYCLIC COMPONENTS
}

\author{
Claudia Chaio ${ }^{1}$ and Piotr Malicki ${ }^{2}$
}

${ }^{1}$ Departamento de Matemática, Facultad de Ciencias Exactas y Naturales, Funes 3350, Universidad Nacional de Mar del Plata, 7600 Mar del Plata, Argentina

${ }^{2}$ Faculty of Mathematics and Computer Science, Nicolaus Copernicus University, Toruń, Poland

We study the degrees of irreducible morphisms between indecomposable modules lying in generalized standard coherent almost cyclic components of Auslander-Reiten quivers of artin algebras.

Key Words: Irreducible morphisms; Radical; Auslander-Reiten quiver; Generalized multicoil.

2000 Mathematics Subject Classification: 16G70, 16G20, 16E10.

\section{INTRODUCTION AND THE MAIN RESULTS}

Throughout this paper, by an algebra is meant an artin algebra over a fixed commutative artin ring $R$, which we shall assume (without loss of generality) to be basic and indecomposable. For an algebra $A$, we denote by $\bmod A$ the category of finitely generated right $A$-modules and by ind $A$ the full subcategory of $\bmod A$ formed by the indecomposable modules. The Jacobson radical $\Re_{A}$ of $\bmod A$ is the ideal generated by all nonisomorphisms between modules in ind $A$, and the infinite radical $\Re_{A}^{\infty}$ of $\bmod A$ is the intersection of all powers $\Re_{A}^{i}, i \geq 1$, of $\Re_{A}$. By a result of Auslander [4], $\Re_{A}^{\infty}=0$ if and only if $A$ is of finite representation type, that is, ind $A$ admits only a finite number of pairwise nonisomorphic modules. On the other hand, if $A$ is of infinite representation type then $\left(\Re_{A}^{\infty}\right)^{2} \neq 0$, by a result proved in [21]. We denote by $\Gamma_{A}$ the Auslander-Reiten quiver of $A$ and by $\tau_{A}=D \operatorname{Tr}$ its translation. We do not distinguish between a module in ind $A$ and the vertex of $\Gamma_{A}$ corresponding to it. By a component of $\Gamma_{A}$ we mean a connected component of the quiver $\Gamma_{A}$. In general, the Auslander-Reiten quiver $\Gamma_{A}$ describes only the quotient category $\bmod A / \Re_{A}^{\infty}$. In order to obtain more information on the structure

Address correspondence to Piotr Malicki, Faculty of Mathematics and Computer Science, Nicolaus Copernicus University, Chopina 12/18, 87-100 Toruń, Poland; Fax: +48-56-622-8979; E-mail: pmalicki@mat.umk.pl 
of a module category mod $A$ it is important to study the behaviour of components of $\Gamma_{A}$ in the module category.

An important research direction towards understanding the structure of module categories is the study of compositions of irreducible morphisms between indecomposable modules. Basic properties of irreducible morphisms between modules and their connection with almost split sequences have been established in the classical papers by Auslander and Reiten [5]-[8] 40 years ago. In [10] Bautista proved that a morphism $f: X \rightarrow Y$ between two indecomposable modules $X$ and $Y$ in a module category $\bmod A$ is irreducible if and only if $f \in \Re_{A}(X, Y) \backslash \Re_{A}^{2}(X, Y)$. This was generalized by Igusa and Todorov [23, Theorem 13.3] who proved that, for a sectional path

$$
X_{0} \stackrel{f_{1}}{\longrightarrow} X_{1} \stackrel{f_{2}}{\longrightarrow} \cdots \stackrel{f_{n-1}}{\longrightarrow} X_{n-1} \stackrel{f_{n}}{\longrightarrow} X_{n}
$$

of irreducible morphisms between indecomposable modules in $\bmod A$, we have $f_{n} \ldots f_{2} f_{1} \in \Re_{A}^{n}\left(X_{0}, X_{n}\right) \backslash \Re_{A}^{n+1}\left(X_{0}, X_{n}\right)$. In [24]-[25] Liu introduced the notions of left and right degrees of irreducible morphisms of modules and showed their importance for describing the shapes of the components of the Auslander-Reiten quivers of algebras of infinite representation type. Let $A$ be an algebra and $f: X \rightarrow Y$ an irreducible morphism in $\bmod A$ with $X$ or $Y$ indecomposable. Following [24] the left degree $d_{l}(f)$ of $f$ is infinite, if for each positive integer $n$, for each module $Z \in \bmod A$ and each morphism $g \in \Re_{A}^{n}(Z, X) \backslash \Re_{A}^{n+1}(Z, X)$ we have that $f g \notin \Re_{A}^{n+2}(Z, Y)$. Otherwise, the left degree of $f$ is the smallest positive integer $m$ such that there exists a morphism $g \in \Re_{A}^{m}(Z, X) \backslash \Re_{A}^{m+1}(Z, X)$ for some module $Z \in \bmod A$ such that $f g \in \Re_{A}^{m+2}(Z, Y)$. Dually, one can define the right degree $d_{r}(f)$ of $f$. The studies of degrees of irreducible morphisms as well as their compositions have attracted recently much attention (see [11]-[20] for some results in this direction).

Following [36], a component $\mathscr{C}$ of $\Gamma_{A}$ is called generalized standard if $\Re_{A}^{\infty}(X, Y)$ $=0$ for all modules $X$ and $Y$ in $\mathscr{C}$. Then, by [36, Theorem 2.3] we know that every generalized standard component $\mathscr{C}$ of $\Gamma_{A}$ is almost periodic, that is, all but finitely many $\tau_{A}$-orbits in $\mathscr{C}$ are periodic. Further, a component $\mathscr{C}$ of $\Gamma_{A}$ is called almost cyclic if all but finitely many modules of $\mathscr{C}$ lie on oriented cycles contained entirely in $\mathscr{C}$. Moreover, a component $\mathscr{C}$ of $\Gamma_{A}$ is called coherent if the following two conditions are satisfied:

(C1) For each projective module $P$ in $\mathscr{C}$ there is an infinite sectional path $P=X_{1} \rightarrow X_{2} \rightarrow \cdots \rightarrow X_{i} \rightarrow X_{i+1} \rightarrow X_{i+2} \rightarrow \cdots$

(C2) For each injective module $I$ in $\mathscr{C}$ there is an infinite sectional path $\cdots \rightarrow Y_{j+2} \rightarrow Y_{j+1} \rightarrow Y_{j} \rightarrow \cdots \rightarrow Y_{2} \rightarrow Y_{1}=I$.

In the paper we study the finiteness of degrees of irreducible morphisms between indecomposable modules lying in generalized standard coherent almost cyclic components of Auslander-Reiten quivers of algebras, plying a prominent role in the representation theory of artin algebras (see [27]-[30], [32]-[33] for their structure and importance). We also note that the class of algebras whose Auslander-Reiten quiver admits generalized standard coherent almost cyclic components is wide and 
contains algebras of arbitrary non-zero, finite or infinite, global dimension. In particular, all multicoil enlargements (see Section 2) of concealed canonical algebras [35], generalized canonical algebras [38], and concealed generalized canonical algebras [31] have this property. The following observation from [24, Lemma 2.2] is relevant for our considerations: for every oriented cycle of irreducible morphisms

$$
X_{0} \stackrel{f_{1}}{\longrightarrow} X_{1} \stackrel{f_{2}}{\longrightarrow} \cdots \stackrel{f_{n-1}}{\longrightarrow} X_{n-1} \stackrel{f_{n}}{\longrightarrow} X_{n}=X_{0}
$$

between indecomposable modules in a module category $\bmod A$, we have $d_{l}\left(f_{i}\right)<\infty$ and $d_{r}\left(f_{j}\right)<\infty$ for some $i, j \in\{1, \ldots, n\}$.

The results presented below are analogous to those obtained in the paper [18]. More precisely, the facts about finiteness of degrees of irreducible morphisms between indecomposable modules lying in coherent almost cyclic Auslander-Reiten components without exceptional configurations of modules (see Section 3 for the definition of these configurations) have been recently proved in [18]. Here, we prove a suitable results for arbitrary generalized standard coherent almost cyclic components of Auslander-Reiten quivers of algebras. We would like to stress that generalized standardness assumption imposed on the considered components is essential (see the proofs of Lemmas 3.3 and 3.4).

The main aim of this paper is to prove the following theorem.

Theorem 1.1. Let $A$ be an algebra, $\mathscr{C}$ a generalized standard coherent almost cyclic component of $\Gamma_{A}$, and $f: X \rightarrow Y$ an irreducible morphism in $\bmod A$ with $X, Y \in \mathscr{C}$. Then the following equivalences hold.

(i) $d_{l}(f)=n$ if and only if there is a sectional path

$$
Z_{0} \stackrel{g_{1}}{\longrightarrow} Z_{1} \stackrel{g_{2}}{\longrightarrow} \cdots \stackrel{g_{n-1}}{\longrightarrow} Z_{n-1} \stackrel{g_{n}}{\longrightarrow} Z_{n}=X
$$

of irreducible morphisms in $\bmod A$ with $Z_{0}, Z_{1}, \ldots, Z_{n-1}$ in $\mathscr{C}$ such that $f g_{n} \ldots g_{1}=0$.

(ii) $d_{r}(f)=n$ if and only if there is a sectional path

$$
Y=U_{0} \stackrel{h_{1}}{\longrightarrow} U_{1} \stackrel{h_{2}}{\longrightarrow} \cdots \stackrel{h_{n-1}}{\longrightarrow} U_{n-1} \stackrel{h_{n}}{\longrightarrow} U_{n}
$$

of irreducible morphisms in $\bmod A$ with $U_{1}, U_{2}, \ldots, U_{n}$ in $\mathscr{C}$ such that $h_{n} \ldots h_{1} f=0$.

As an immediate consequence of the proof of Theorem 1.1 we obtain the following facts.

Corollary 1.2. Let $A$ be an algebra, $\mathscr{C}$ a generalized standard coherent almost cyclic component of $\Gamma_{A}$, and $f: X \rightarrow Y$ an irreducible morphism in $\bmod A$ with $X, Y \in \mathscr{C}$. Then the following statements hold.

(i) If $d_{l}(f)<\infty$, then $f$ is an epimorphism. 
(ii) If $d_{r}(f)<\infty$, then $f$ is a monomorphism.

(iii) If $d_{l}(f)<\infty$, then $d_{r}(f)=\infty$.

(iv) If $d_{r}(f)<\infty$, then $d_{l}(f)=\infty$.

Moreover, from the proof of Theorem 1.1 and [23, Theorem 13.3] we receive the following fact.

Corollary 1.3. Let $A$ be an algebra, $\mathscr{C}$ a generalized standard coherent almost cyclic component of $\Gamma_{A}, f: X \rightarrow Y$ an irreducible morphism in $\bmod A$ with $X, Y \in$ $\mathscr{C}$, and $n$ a positive integer. Then

(i) $d_{l}(f)=n$ if and only if the inclusion morphism $\iota: \operatorname{Ker} f \rightarrow X$ is such that $\iota \in \Re_{A}^{n}(\operatorname{Ker} f, X) \backslash \Re_{A}^{n+1}(\operatorname{Ker} f, X)$.

(ii) $d_{r}(f)=n$ if and only if the quotient morphism $\theta: Y \rightarrow$ Coker $f$ is such that $\theta \in \Re_{A}^{n}(Y, \operatorname{Coker} f) \backslash \Re_{A}^{n+1}(Y$, Coker $f)$.

For basic background on the representation theory of algebras we refer to the books [1], [9] and [34]. We refer the reader to [24] for a detailed account on the degrees of irreducible morphisms.

\section{GENERALIZED STANDARD COHERENT ALMOST CYCLIC COMPONENTS}

Let $A$ be an algebra. By a component of $\Gamma_{A}$ we mean a connected component of $\Gamma_{A}$. A component in $\Gamma_{A}$ of the form $\mathbb{Z} \mathbb{A}_{\infty} /\left(\tau^{r}\right), r \geq 1$, is said to be a stable tube of rank $r$. Therefore, a stable tube of rank $r$ in $\Gamma_{A}$ is an infinite component consisting of $\tau_{A}$-periodic indecomposable $A$-modules having period $r$. A stable tube of rank 1 is said to be homogeneous. Moreover, a stable tube $\mathscr{T}$ of $\Gamma_{A}$ admits a distinguished $\tau_{A}$-orbit, called the mouth of $\mathscr{T}$, consisting of modules having exactly one direct predecessor and exactly one direct successor in $\mathscr{T}$. An indecomposable module $X$ is called a brick if its endomorphism algebra $\operatorname{End}_{A}(X)$ is a division algebra. We also note that the division algebras of all modules $X$ lying on the mouth of a generalized standard stable tube of $\Gamma_{A}$ are isomorphic. For a component $\mathscr{C}$ of $\Gamma_{A}$ we denote by $\operatorname{ann}_{A}(\mathscr{C})$ the annihilator of $\mathscr{C}$ in $A$, that is, the intersection of the annihilators $\operatorname{ann}_{A}(X)$ of all modules $X$ in $\mathscr{C}$. If $\operatorname{ann}_{A}(\mathscr{C})=0$, then $\mathscr{C}$ is called faithful. Finally, two components $\mathscr{C}$ and $\mathscr{D}$ of $\Gamma_{A}$ are called orthogonal if $\operatorname{Hom}_{A}(X, Y)=0$ and $\operatorname{Hom}_{A}(Y, X)=0$ for all modules $X$ in $\mathscr{C}$ and $Y$ in $\mathscr{D}$.

The following characterization of generalized standard stable tubes of an Auslander-Reiten quiver has been established in [36, Corollary 5.3] (see also [37, Lemma 3.1]).

Proposition 2.1. Let $A$ be an algebra and $\mathscr{T}$ a stable tube of $\Gamma_{A}$. The following statements are equivalent. 
(i) $\mathscr{T}$ is generalized standard.

(ii) The mouth of $\mathscr{T}$ consists of pairwise orthogonal bricks.

(iii) $\Re_{A}^{\infty}(X, X)=0$ for any module $X$ in $\mathscr{T}$.

The fundamental role in the proofs of our main results will be played by the following characterization of coherent almost cyclic Auslander-Reiten components proved in $[28$, Theorem A].

Theorem 2.2. Let $A$ be an algebra and $\mathscr{C}$ a component of $\Gamma_{A}$. The following conditions are equivalent.

(i) $\mathscr{C}$ is coherent and almost cyclic.

(ii) $\mathscr{C}$ is a generalized multicoil.

The main aim of this section is to recall the concepts of generalized multicoils and generalized multicoil enlargements of algebras from [29, Section 3].

We start with the concepts of one-point extensions and one-point coextensions of algebras. Let $A$ be an algebra, let $F$ be a division algebra over $R$, and let $M={ }_{F} M_{A}$ be an $F$-A-bimodule such that $M_{A} \in \bmod A$ and $R$ acts centrally on ${ }_{F} M_{A}$. Then the one-point extension of $A$ by $M$ is the matrix artin $R$-algebra of the form

$$
A[M]=\left[\begin{array}{cc}
A & 0 \\
F M_{A} & F
\end{array}\right]=\left\{\left[\begin{array}{cc}
a & 0 \\
m & f
\end{array}\right] ; f \in F, a \in A, m \in M\right\}
$$

with the usual addition and multiplication. Then the valued quiver $Q_{A[M]}$ of $A[M]$ contains the valued quiver $Q_{A}$ of $A$ as a convex subquiver, and there is an additional (extension) vertex which is a source. We may identify the category $\bmod A[M]$ with the category whose objects are triples $(V, X, \varphi)$, where $X \in \bmod A$, $V \in \bmod F$, and $\varphi: V_{F} \rightarrow \operatorname{Hom}_{A}(M, X)_{F}$ is an $F$-linear map. $A$ morphism $h:(V, X, \varphi) \rightarrow(W, Y, \psi)$ is given by a pair $(f, g)$, where $f: V \rightarrow W$ is $F$-linear, $g: X \rightarrow Y$ is a morphism in $\bmod A$ and $\psi f=\operatorname{Hom}_{A}(M, g) \varphi$. Then the new indecomposable projective $A[M]$-module $P$ is given by the triple $(F, M, \bullet)$ where - : $F_{F} \rightarrow \operatorname{Hom}_{A}(M, M)_{F}$ assigns to the identity element of $F$ the identity morphism of $M$. An important class of such one-point extensions occurs in the following situation. Let $\Lambda$ be a basic artin $R$-algebra, $P$ an indecomposable projective $\Lambda$-module, ${ }_{\Lambda} \Lambda=P \oplus Q$, and assume that $\operatorname{Hom}_{\Lambda}(P, Q \oplus \operatorname{rad} P)=0$. Since $P$ is indecomposable projective, $S=P / \operatorname{rad} P$ is a simple $\Lambda$-module and hence $\operatorname{End}_{\Lambda}(S)$ is a division algebra. Moreover, the canonical homomorphism of algebras $\operatorname{End}_{\Lambda}(P) \rightarrow \operatorname{End}_{\Lambda}(S)$ is an isomorphism. Then we obtain isomorphisms of algebras

$$
\Lambda \cong \operatorname{End}_{\Lambda}\left(\Lambda_{\Lambda}\right) \cong\left[\begin{array}{cc}
A & 0 \\
F & M_{A}
\end{array}\right]=A[M]
$$

where $F=\operatorname{End}_{\Lambda}(P), A=\operatorname{End}_{\Lambda}(Q)$, and $M={ }_{F} M_{A}=\operatorname{Hom}_{\Lambda}(Q, P) \cong \operatorname{rad} P$. Clearly $R$ acts centrally on ${ }_{F} M_{A}$. We note that if the valued quiver of an artin 
algebra $\Lambda$ has no oriented cycles then $\Lambda$ can be obtained from a semisimple algebra by a sequence of one-point extensions of the above form.

Dually, one defines also the one-point coextension of $A$ by ${ }_{F} M_{A}$ as the matrix algebra

$$
[M] A=\left[\begin{array}{cc}
F & 0 \\
D\left({ }_{F} M_{A}\right) & A
\end{array}\right] .
$$

For each bimodule ${ }_{F} M_{A}$ considered in the paper we assume that $A$ is an algebra, $M_{A} \in \bmod A, F$ is a division algebra, and $R$ acts centrally on ${ }_{F} M_{A}$.

A generalized multicoil is a translation quiver constructed inductively from a stable tube by a sequence of operation called admissible. We recall briefly the notion of admissible operations (see [28], [29]).

For a division algebra $F$ and $r \geq 1$, we denote by $T_{r}(F)$ the $r \times r$-lower triangular matrix algebra

$$
\left[\begin{array}{cccccc}
F & 0 & 0 & \ldots & 0 & 0 \\
F & F & 0 & \ldots & 0 & 0 \\
F & F & F & \ldots & 0 & 0 \\
\vdots & \vdots & \vdots & \ddots & \vdots & \vdots \\
F & F & F & \ldots & F & 0 \\
F & F & F & \ldots & F & F
\end{array}\right]
$$

Given a generalized standard component $\Gamma$ of $\Gamma_{A}$, and an indecomposable module $X$ in $\Gamma$, the support $\mathcal{S}(X)$ of the functor $\left.\operatorname{Hom}_{A}(X,-)\right|_{\Gamma}$ is the $R$-linear category defined as follows [3]. Let $\mathcal{H}_{X}$ denote the full subcategory of $\Gamma$ consisting of the indecomposable modules $M$ in $\Gamma$ such that $\operatorname{Hom}_{A}(X, M) \neq 0$, and $\mathcal{I}_{X}$ denote the ideal of $\mathcal{H}_{X}$ consisting of the morphisms $f: M \rightarrow N$ (with $M, N$ in $\mathcal{H}_{X}$ ) such that $\operatorname{Hom}_{A}(X, f)=0$. We define $\mathcal{S}(X)$ to be the quotient category $\mathcal{H}_{X} / \mathcal{I}_{X}$. Following the above convention, we usually identify the $R$-linear category $\mathcal{S}(X)$ with its quiver.

From now on, we let $A$ be an algebra and $\Gamma$ a generalized standard component of $\Gamma_{A}$. We define five admissible operations and their duals modifying the translation quiver $\Gamma=(\Gamma, \tau)$ to a new translation quiver $\left(\Gamma^{\prime}, \tau^{\prime}\right)$ and the algebra $A$ to a new algebra $A^{\prime}$, depending on the shape of the support $\mathcal{S}(X)$ (see [28, Section 2] or [18, Section 2] for the figures illustrating the modified translation quivers $\left.\Gamma^{\prime}\right)$. In the considerations below $X$ is a brick, which is called a pivot, and $F=F_{X}=\operatorname{End}_{A}(X)$ is the division algebra associated to $X$.

(ad 1) Assume $\mathcal{S}(X)$ is an infinite sectional path starting at $X$ :

$$
X=X_{0} \rightarrow X_{1} \rightarrow X_{2} \rightarrow \cdots
$$

In this case, we let $t \geq 1$ be a positive integer, $D=T_{t}(F)$ and $Y_{1}, Y_{2}, \ldots, Y_{t}$ denote the indecomposable injective $D$-modules with $Y=Y_{1}$ the unique indecomposable projective-injective $D$-module. We define the modified algebra $A^{\prime}$ of $A$ to be the one-point extension

$$
A^{\prime}=(A \times D)[X \oplus Y]
$$


and the modified translation quiver $\Gamma^{\prime}$ of $\Gamma$ to be obtained by adding to $\Gamma$ the quiver $\Gamma_{D}$ and the rectangle consisting of the modules

$$
Z_{i j}=\left(F, X_{i} \oplus Y_{j},\left[\begin{array}{l}
1 \\
1
\end{array}\right]\right) \text { for } i \geq 0,1 \leq j \leq t,
$$

and $X_{i}^{\prime}=\left(F, X_{i}, 1\right)$ for $i \geq 0$. The translation $\tau^{\prime}$ of $\Gamma^{\prime}$ is defined as follows: $\tau^{\prime} Z_{i j}=Z_{i-1, j-1}$ if $i \geq 1, j \geq 2, \tau^{\prime} Z_{i 1}=X_{i-1}$ if $i \geq 1, \tau^{\prime} Z_{0 j}=Y_{j-1}$ if $j \geq 2, Z_{01}$ is projective, $\tau^{\prime} X_{0}^{\prime}=Y_{t}, \tau^{\prime} X_{i}^{\prime}=Z_{i-1, t}$ if $i \geq 1, \tau^{\prime}\left(\tau^{-1} X_{i}\right)=X_{i}^{\prime}$ provided $X_{i}$ is not an injective $A$-module, otherwise $X_{i}^{\prime}$ is injective in $\Gamma^{\prime}$. For the remaining vertices of $\Gamma^{\prime}, \tau^{\prime}$ coincides with the translation of $\Gamma$, or $\Gamma_{D}$, respectively.

If $t=0$ we define the modified algebra $A^{\prime}$ to be the one-point extension $A^{\prime}=$ $A[X]$ and the modified translation quiver $\Gamma^{\prime}$ to be the translation quiver obtained from $\Gamma$ by inserting only the sectional path consisting of the vertices $X_{i}^{\prime}, i \geq 0$.

Since $\Gamma$ is a generalized standard component of $\Gamma_{A}$, we then have that $\Gamma^{\prime}$ is a generalized standard component of $\Gamma_{A^{\prime}}$.

In case $\Gamma$ is a stable tube, it is clear that any module on the mouth of $\Gamma$ satisfies the condition for being a pivot for the above operation. Actually, the above operation is, in this case, the tube insertion as considered in [22].

(ad 2) Suppose that $\mathcal{S}(X)$ consists of two sectional paths starting at $X$, one infinite and the other finite with at least one arrow:

$$
Y_{t} \leftarrow \cdots \leftarrow Y_{2} \leftarrow Y_{1} \leftarrow X=X_{0} \rightarrow X_{1} \rightarrow X_{2} \rightarrow \cdots
$$

where $t \geq 1$. In particular, $X$ is necessarily injective. We define the modified algebra $A^{\prime}$ of $A$ to be the one-point extension $A^{\prime}=A[X]$ and the modified translation quiver $\Gamma^{\prime}$ of $\Gamma$ to be obtained by inserting in $\Gamma$ the rectangle consisting of the modules

$$
Z_{i j}=\left(F, X_{i} \oplus Y_{j},\left[\begin{array}{l}
1 \\
1
\end{array}\right]\right) \text { for } i \geq 1,
$$

$1 \leq j \leq t$, and $X_{i}^{\prime}=\left(F, X_{i}, 1\right)$ for $i \geq 1$. The translation $\tau^{\prime}$ of $\Gamma^{\prime}$ is defined as follows: $X_{0}^{\prime}$ is projective-injective, $\tau^{\prime} Z_{i j}=Z_{i-1, j-1}$ if $i \geq 2, j \geq 2, \tau^{\prime} Z_{i 1}=X_{i-1}$ if $i \geq 1, \tau^{\prime} Z_{1 j}=Y_{j-1}$ if $j \geq 2, \tau^{\prime} X_{i}^{\prime}=Z_{i-1, t}$ if $i \geq 2, \tau^{\prime} X_{1}^{\prime}=Y_{t}, \tau^{\prime}\left(\tau^{-1} X_{i}\right)=X_{i}^{\prime}$ provided $X_{i}$ is not an injective $A$-module, otherwise $X_{i}^{\prime}$ is injective in $\Gamma^{\prime}$. For the remaining vertices of $\Gamma^{\prime}, \tau^{\prime}$ coincides with the translation $\tau$ of $\Gamma$.

Since $\Gamma$ is a generalized standard component of $\Gamma_{A}$, we then have that $\Gamma^{\prime}$ is a generalized standard component of $\Gamma_{A^{\prime}}$.

(ad 3) Assume $\mathcal{S}(X)$ is the mesh-category of two parallel sectional paths:

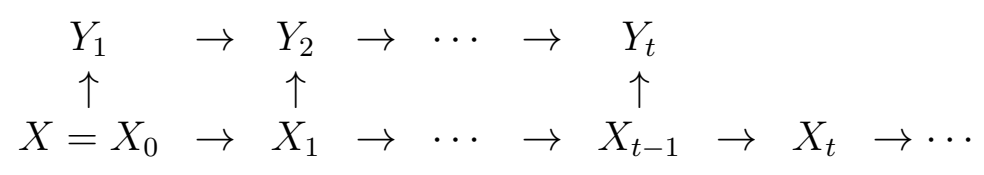

where $t \geq 2$. In particular, $X_{t-1}$ is necessarily injective. Moreover, we consider the translation subquiver $\bar{\Gamma}$ of $\Gamma$ obtained by deleting the arrows $Y_{i} \rightarrow \tau_{A}^{-1} Y_{i-1}$. 
We assume that the union $\widehat{\Gamma}$ of connected components of $\bar{\Gamma}$ containing the vertices $\tau_{A}^{-1} Y_{i-1}, 2 \leq i \leq t$, is a finite translation quiver. Then $\bar{\Gamma}$ is a disjoint union of $\widehat{\Gamma}$ and a cofinite full translation subquiver $\Gamma^{*}$, containing the pivot $X$. We define the modified algebra $A^{\prime}$ of $A$ to be the one-point extension $A^{\prime}=A[X]$ and the modified translation quiver $\Gamma^{\prime}$ of $\Gamma$ to be obtained from $\Gamma^{*}$ by inserting the rectangle consisting of the modules

$$
Z_{i j}=\left(F, X_{i} \oplus Y_{j},\left[\begin{array}{l}
1 \\
1
\end{array}\right]\right) \text { for } i \geq j, 1 \leq j \leq t,
$$

and $X_{i}^{\prime}=\left(F, X_{i}, 1\right)$ for $i \geq 1$. The translation $\tau^{\prime}$ of $\Gamma^{\prime}$ is defined as follows: $X_{0}^{\prime}$ is projective, $\tau^{\prime} Z_{i j}=Z_{i-1, j-1}$ if $i \geq j, 2 \leq j \leq t, \tau^{\prime} Z_{i 1}=X_{i-1}$ if $i \geq 1, \tau^{\prime} X_{i}^{\prime}=Y_{i}$ if $1 \leq i \leq t, \tau^{\prime} X_{i}^{\prime}=Z_{i-1, t}$ if $i \geq t+1, \tau^{\prime} Y_{j}=X_{j-2}^{\prime}$ if $2 \leq j \leq t, \tau^{\prime}\left(\tau^{-1} X_{i}\right)=X_{i}^{\prime}$, if $i \geq t$ provided $X_{i}$ is not injective in $\Gamma$, otherwise $X_{i}^{\prime}$ is injective in $\Gamma^{\prime}$. For the remaining vertices of $\Gamma^{\prime}, \tau^{\prime}$ coincides with the translation $\tau$ of $\Gamma^{*}$. We note that $X_{t-1}^{\prime}$ is injective.

Since $\Gamma$ is a generalized standard component of $\Gamma_{A}$, we then have that $\Gamma^{\prime}$ is a generalized standard component of $\Gamma_{A^{\prime}}$.

(ad 4) Suppose that $\mathcal{S}(X)$ is an infinite sectional path starting at $X$

$$
X=X_{0} \rightarrow X_{1} \rightarrow X_{2} \rightarrow \cdots .
$$

Moreover, we choose $Y$ in $\Gamma$ such that $\mathcal{S}(Y)$ is a finite sectional path starting at $Y$

$$
Y=Y_{1} \rightarrow Y_{2} \rightarrow \cdots \rightarrow Y_{t}
$$

where $t \geq 1, \mathcal{S}(X)$ and $\mathcal{S}(Y)$ are disjoint, and $F_{Y}=F=F_{X}$. Let $r$ be a positive integer. Moreover, we consider the translation subquiver $\bar{\Gamma}$ of $\Gamma$ obtained by deleting the arrows $Y_{i} \rightarrow \tau_{A}^{-1} Y_{i-1}$. We assume that the union $\widehat{\Gamma}$ of connected components of $\bar{\Gamma}$ containing the vertices $\tau_{A}^{-1} Y_{i-1}, 2 \leq i \leq t$, is a finite translation quiver. Then $\bar{\Gamma}$ is a disjoint union of $\widehat{\Gamma}$ and a cofinite full translation subquiver $\Gamma^{*}$, containing the pivot $X$. For $r=0$ we define the modified algebra $A^{\prime}$ of $A$ to be the one-point extension $A^{\prime}=A[X \oplus Y]$ and the modified translation quiver $\Gamma^{\prime}$ of $\Gamma$ to be obtained from $\Gamma^{*}$ by inserting the rectangle consisting of the modules

$$
Z_{i j}=\left(F, X_{i} \oplus Y_{j},\left[\begin{array}{l}
1 \\
1
\end{array}\right]\right) \text { for } i \geq 0,1 \leq j \leq t,
$$

and $X_{i}^{\prime}=\left(F, X_{i}, 1\right)$ for $i \geq 1$. The translation $\tau^{\prime}$ of $\Gamma^{\prime}$ is defined as follows: $\tau^{\prime} Z_{i j}=Z_{i-1, j-1}$ if $i \geq 1, j \geq 2, \tau^{\prime} Z_{i 1}=X_{i-1}$ if $i \geq 1, \tau^{\prime} Z_{0 j}=Y_{j-1}$ if $j \geq 2, Z_{01}$ is projective, $\tau^{\prime} X_{0}^{\prime}=Y_{t}, \tau^{\prime} X_{i}^{\prime}=Z_{i-1, t}$ if $i \geq 1, \tau^{\prime}\left(\tau^{-1} X_{i}\right)=X_{i}^{\prime}$ provided $X_{i}$ is not injective in $\Gamma$, otherwise $X_{i}^{\prime}$ is injective in $\Gamma^{\prime}$. For the remaining vertices of $\Gamma^{\prime}, \tau^{\prime}$ coincides with the translation of $\Gamma^{*}$. 
For $r \geq 1$, we define the modified algebra $A^{\prime}$ of $A$ to be the triangular matrix algebra of the form:

$$
A^{\prime}=\left[\begin{array}{cccccc}
A & 0 & 0 & \ldots & 0 & 0 \\
Y & F & 0 & \ldots & 0 & 0 \\
Y & F & F & \ldots & 0 & 0 \\
\vdots & \vdots & \vdots & \ddots & \vdots & \vdots \\
Y & F & F & \ldots & F & 0 \\
X \oplus Y & F & F & \ldots & F & F
\end{array}\right]
$$

with $r+2$ columns and rows and the modified translation quiver $\Gamma^{\prime}$ of $\Gamma$ to be obtained from $\Gamma^{*}$ by inserting the following modules

$$
\begin{aligned}
& U_{s l}= \begin{cases}\left(F, Y_{l}, 1\right) & \text { for } s=1,1 \leq l \leq t, \\
\left(F, U_{s, l-1}, 1\right) & \text { for } 2 \leq s \leq r, 1 \leq l<t+s, \\
(F, 0,0) & \text { for } 2 \leq s \leq r, l=t+s\end{cases} \\
& Z_{i j}=\left(F, X_{i} \oplus U_{r j},\left[\begin{array}{l}
1 \\
1
\end{array}\right]\right) \text { for } i \geq 0,1 \leq j \leq t+r,
\end{aligned}
$$

and $X_{i}^{\prime}=\left(F, X_{i}, 1\right)$ for $i \geq 0$. In the above formulas $U_{s l}$ is treated as a module over the algebra $A_{s}=A_{s-1}\left[U_{s-1,1}\right]$, where $A_{0}=A$ and $U_{01}=Y$ (in other words $A_{s}$ is an algebra consisting of matrices obtained from the matrices belonging to $A^{\prime}$ by choosing the first $s+1$ rows and columns). The translation $\tau^{\prime}$ of $\Gamma^{\prime}$ is defined as follows: $\tau^{\prime} Z_{i j}=Z_{i-1, j-1}$ if $i \geq 1, j \geq 2, \tau^{\prime} Z_{i 1}=X_{i-1}$ if $i \geq 1, \tau^{\prime} Z_{0 j}=U_{r, j-1}$ if $2 \leq j \leq t+r, Z_{01}, U_{s 1}, 1 \leq s \leq r$ are projective, $\tau^{\prime} U_{s l}=U_{s-1, l-1}$ if $2 \leq s \leq r$, $2 \leq l \leq t+r, \tau^{\prime} U_{1 l}=Y_{l-1}$ if $2 \leq l \leq t+1, \tau^{\prime} X_{0}^{\prime}=U_{r, t+r}, \tau^{\prime} X_{i}^{\prime}=Z_{i-1, t+r}$ if $i \geq 1, \tau^{\prime}\left(\tau^{-1} X_{i}\right)=X_{i}^{\prime}$ provided $X_{i}$ is not injective in $\Gamma$, otherwise $X_{i}^{\prime}$ is injective in $\Gamma^{\prime}$. For the remaining vertices of $\Gamma^{\prime}, \tau^{\prime}$ coincides with the translation of $\Gamma^{*}$, or $\Gamma_{G}$, respectively.

We note that if $r \geq 1$, then the quiver $Q_{A^{\prime}}$ of $A^{\prime}$ is obtained from the quiver of the double one-point extension $A[X][Y]$ by adding a path of length $r+1$ with source at the extension vertex of $A[X]$ and sink at the extension vertex of $A[Y]$.

Since $\Gamma$ is a generalized standard component of $\Gamma_{A}$, we then have that $\Gamma^{\prime}$ is a generalized standard component of $\Gamma_{A^{\prime}}$.

To the definition of the next admissible operation we need also the finite versions of the admissible operations ( $\operatorname{ad} 1),(\operatorname{ad} 2),(\operatorname{ad} 3),(\operatorname{ad} 4)$, which we noticed by (fad 1), (fad 2), (fad 3) and (fad 4), respectively. In order to obtain this operations all infinite sectional paths of the form $X_{0} \rightarrow X_{1} \rightarrow X_{2} \rightarrow \cdots$ (in the definitions of $(\operatorname{ad} 1),(\operatorname{ad} 2),(\operatorname{ad} 3),(\operatorname{ad} 4))$ we replace by the finite sectional paths of the form $X_{0} \rightarrow X_{1} \rightarrow X_{2} \rightarrow \cdots \rightarrow X_{s}$. For the operation (fad 1) $s \geq 0$, for (fad 2) and (fad 4) $s \geq 1$, and for (fad 3) $s \geq t-1$. In all above operations $X_{s}$ is injective (see [28] or [29] for the details).

(ad 5) We define the modified algebra $A^{\prime}$ of $A$ to be the iteration of the extensions described in the definitions of the admissible operations ( $\operatorname{ad} 1),(\operatorname{ad} 2)$, 
(ad 3), (ad 4), and their finite versions corresponding to the operations (fad 1), (fad 2), (fad 3) and (fad 4). The modified translation quiver $\Gamma^{\prime}$ of $\Gamma$ is obtained in the following three steps: first we are doing on $\Gamma$ one of the operations (fad 1$)$, (fad 2) or (fad 3), next a finite number (possibly empty) of the operation (fad 4) and finally the operation ( $\mathrm{ad}$ ), and in such a way that the new projective vertices lie on a common infinite sectional path.

Since $\Gamma$ is a generalized standard component of $\Gamma_{A}$, we then have that $\Gamma^{\prime}$ is a generalized standard component of $\Gamma_{A^{\prime}}$.

Finally, together with each of the admissible operations $(\operatorname{ad} 1),(\operatorname{ad} 2),(\operatorname{ad} 3)$, $(\operatorname{ad} 4)$ and $(\operatorname{ad} 5)$, we consider its dual, denoted by $\left(\operatorname{ad} 1^{*}\right),\left(\operatorname{ad} 2^{*}\right),\left(\operatorname{ad} 3^{*}\right),\left(\operatorname{ad} 4^{*}\right)$ and $\left(\operatorname{ad} 5^{*}\right)$. These ten operations are called the admissible operations.

Clearly, the admissible operations can be defined as operations on translation quivers rather than on Auslander-Reiten components. The definitions are done in the obvious manner (see [28] for the details).

A connected translation quiver $\Gamma$ is said to be a generalized multicoil [28] if $\Gamma$ can be obtained from a finite family $\mathcal{T}_{1}, \mathcal{T}_{2}, \ldots, \mathcal{T}_{s}$ of stable tubes by an iterated application of admissible operations $(\operatorname{ad} 1),\left(\operatorname{ad} 1^{*}\right),(\operatorname{ad} 2),\left(\operatorname{ad} 2^{*}\right),(\operatorname{ad} 3),\left(\operatorname{ad} 3^{*}\right)$, $(\operatorname{ad} 4),\left(\operatorname{ad} 4^{*}\right),(\operatorname{ad} 5)$ or $\left(\operatorname{ad} 5^{*}\right)$. If $s=1$, such a translation quiver $\Gamma$ is said to be a generalized coil. The admissible operations of types ( $\operatorname{ad} 1),(\operatorname{ad} 2),(\operatorname{ad} 3)$, $\left(\operatorname{ad~} 1^{*}\right),\left(\operatorname{ad~} 2^{*}\right)$ and $\left(\operatorname{ad} 3^{*}\right)$ have been introduced in [2], [3], and the admissible operations (ad 4) and $\left(\operatorname{ad~} 4^{*}\right)$ for $r=0$ in [26]. We refer also [28, Section 2], [33, Section 4] or [18, Section 2] for the examples of generalized multicoils.

Finally, let $C$ be a (not necessarily indecomposable) algebra and $\mathscr{T}^{C}$ a family of pairwise orthogonal generalized standard stable tubes of $\Gamma_{C}$. We say that an algebra $A$ is a generalized multicoil enlargement of $C$ using modules from $\mathscr{T}^{C}$ if $A$ is obtained from $C$ by an iteration of admissible operations of types $(\operatorname{ad} 1)-(\operatorname{ad} 5)$ and $\left(\operatorname{ad} 1^{*}\right)-\left(\operatorname{ad} 5^{*}\right)$ performed either on stable tubes of $\mathscr{T}^{C}$, or on generalized multicoils obtained from stable tubes of $\mathscr{T}^{C}$ by means of operations done so far. Note that $C$ is a quotient algebra of $A$.

The following theorem follows from Proposition 2.1 and the proof of Theorem $\mathrm{A}$ in $[29]$.

Theorem 2.3. Let $A$ be an algebra, $\mathscr{C}$ be a component of $\Gamma_{A}$, and $B=A / \operatorname{ann}_{A}(\mathscr{C})$. Then the following statements are equivalent:

(i) $\mathscr{C}$ is generalized standard and a generalized multicoil.

(ii) $B$ is a generalized multicoil enlargement of an algebra $C$ using modules from a generalized standard family $\mathscr{T}^{C}$ of stable tubes of $\Gamma_{C}$ and $\mathscr{C}$ is the generalized standard multicoil obtained from $\mathscr{T}^{C}$ by the translation quiver admissible operations corresponding to the algebra admissible operations leading from $C$ to $B$. 


\section{PRELIMINARY RESULTS}

Let $A$ be an algebra, $X, Y$ be the indecomposable modules in $\bmod A$, and $f$ : $X \rightarrow Y$ be an irreducible morphism in $\bmod A$. If $Y$ is not projective, denote by $\epsilon(Y)$ the almost split sequence ending at $Y$ and by $\alpha(f)$ the number of indecomposable direct summands of the middle term of $\epsilon(Y)$. Dually, if $X$ is not injective, we shall denote by $\epsilon^{\prime}(X)$ the almost split sequence starting at $X$ and by $\alpha^{\prime}(f)$ the number of indecomposable direct summands of the middle term of $\epsilon^{\prime}(X)$.

Let $A$ be an algebra and $\mathscr{C}$ a generalized standard coherent almost cyclic component of $\Gamma_{A}$. We need the following notion. A vertex $U$ of $\mathscr{C}$ is said to belong to the mouth of $\mathscr{C}$ if $U$ has at most one immediate predecessor or at most one immediate successor. We note that any arrow $U \stackrel{\omega}{\longrightarrow} V$ in $\mathscr{C}$ is pointing to infinity (i.e. there is an infinite sectional path $U \stackrel{\omega}{\longrightarrow} V \longrightarrow \cdots)$ or is pointing to the mouth (i.e. there is a finite sectional path $U \stackrel{\omega}{\longrightarrow} V \longrightarrow \cdots \longrightarrow W$ with $W$ belonging to the mouth of $\mathscr{C}$ ). Moreover, any generalized multicoil has only three types of arrows: arrows pointing only to infinity, arrows pointing only to the mouth and arrows pointing to infinity and to the mouth (see [28, Example], [33, Example 4.2] or [18, Example 2.2]).

Following [18], a full translation subquiver of $\Gamma_{A}$ of one of the forms

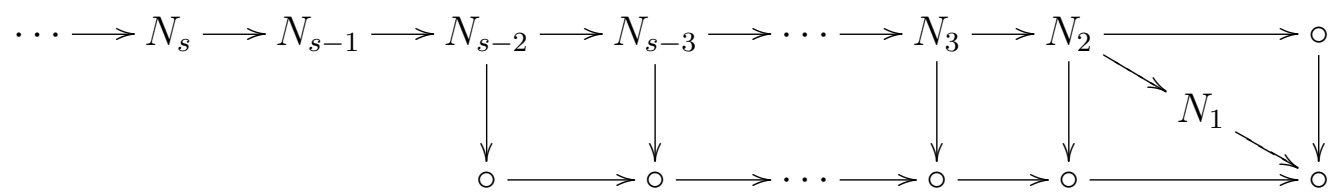

or

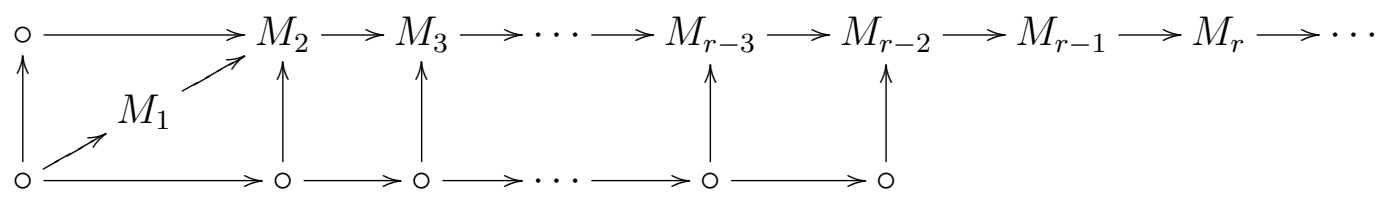

where $N_{1}$ and $N_{s}$ are injective, $M_{1}$ and $M_{r}$ are projective, $s, r \geq 4$, and

$$
\cdots \rightarrow N_{s} \rightarrow N_{s-1} \rightarrow \cdots \rightarrow N_{2} \rightarrow N_{1}, \quad M_{1} \rightarrow M_{2} \rightarrow \cdots \rightarrow M_{r-1} \rightarrow M_{r} \rightarrow \cdots
$$

are the infinite sectional paths in $\Gamma_{A}$, is said to be an exceptional configuration of modules.

In the proof of Lemma 3.3 we shall need the following useful fact (see [18, Lemma 3.2]).

Lemma 3.1. Let $A$ be an algebra, $s$ a positive integer, and $f: X \rightarrow Y$ be an irreducible morphism in $\bmod A$. Let $\mathscr{C}$ be a component of $\Gamma_{A}$ which admits a meshcomplete full translation subquiver of the form

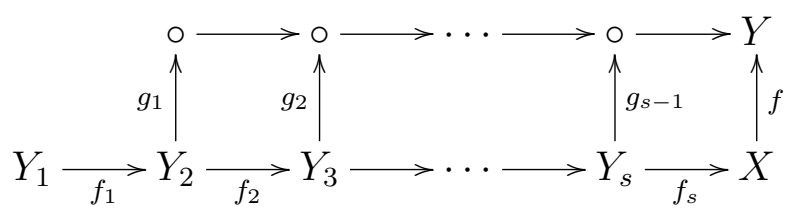


where $Y_{1} \stackrel{f_{1}}{\longrightarrow} Y_{2} \stackrel{f_{2}}{\longrightarrow} \cdots \stackrel{f_{s-1}}{\longrightarrow} Y_{s} \stackrel{f_{s}}{\longrightarrow} X$ is a sectional path of irreducible morphisms in $\bmod A$ such that $f f_{s} \ldots f_{1}=0$ and $\alpha\left(g_{1}\right)=1$. Then $d_{l}(f)=s$, and $d_{l}\left(g_{i}\right)=i$ for $i=1, \ldots, s-1$.

We shall need also the following direct consequence of lemma stated in [2, (2.1)].

Lemma 3.2. Let $A$ be an algebra and

$$
\begin{gathered}
0 \rightarrow M_{1} \stackrel{\left[f_{1}, u_{1}\right]^{t}}{\longrightarrow} N_{1} \oplus M_{2} \stackrel{\left[u_{2}, f_{2}\right]}{\longrightarrow} N_{2} \rightarrow 0, \\
0 \rightarrow M_{2} \stackrel{\left[f_{2}, v_{1}\right]^{t}}{\longrightarrow} N_{2} \oplus M_{3} \stackrel{\left[v_{2}, f_{3}\right]}{\longrightarrow} N_{3} \rightarrow 0
\end{gathered}
$$

be short exact sequences in $\bmod A$. Then the sequence

$$
0 \rightarrow M_{1} \stackrel{\left[f_{1}, v_{1} u_{1}\right]^{t}}{\longrightarrow} N_{1} \oplus M_{3} \stackrel{\left[-v_{2} u_{2}, f_{3}\right]}{\longrightarrow} N_{3} \rightarrow 0
$$

is exact.

Lemma 3.3. Let $A$ be an algebra and $\mathscr{C}$ a generalized standard coherent almost cyclic component of $\Gamma_{A}$ with an exceptional configuration of modules of one of the forms

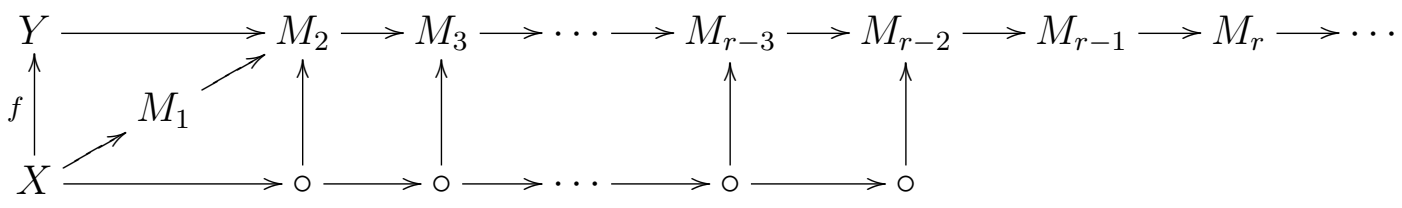

or

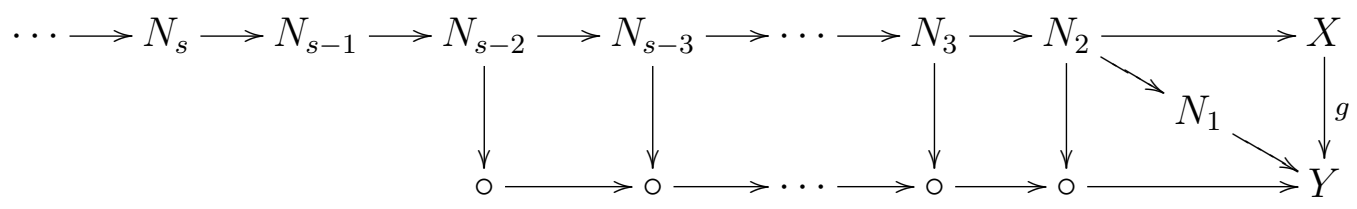

Then

(i) $d_{r}(f)=\infty$ and $d_{l}(f)=m$ for some integer $m \geq 1$.

(ii) $d_{l}(g)=\infty$ and $d_{r}(g)=n$ for some integer $n \geq 1$.

Proof. We shall prove only (i), because the proof of (ii) is dual. Let $\mathscr{C}$ be a generalized standard coherent almost cyclic component of $\Gamma_{A}$. Consider the quotient algebra $B=A / \operatorname{ann}_{A}(\mathscr{C})$. Then $\mathscr{C}$ is a generalized standard component of $\Gamma_{B}$. Further, it follows from [39, Theorem 1] that the additive category $\operatorname{add}(\mathscr{C})$ of $\mathscr{C}$ is closed under extensions in $\bmod A$, and hence also in $\bmod B$. Then for every indecomposable module $M$ in $\mathscr{C}$ we have an isomorphism $\operatorname{Ext}_{A}^{1}(M, M) \cong \operatorname{Ext}_{B}^{1}(M, M)$, and the equality $\operatorname{End}_{A}(M)=\operatorname{End}_{B}(M)$, because $M$ is a $B$-module. Therefore, we may assume that $\operatorname{ann}_{A}(\mathscr{C})=0$, that is, $\mathscr{C}$ is a faithful component of $\Gamma_{A}$. Then 
it follows from Theorem 2.3 that there is an algebra $C$ (not necessarily indecomposable) and a family $\mathscr{T}_{1}, \mathscr{T}_{2}, \ldots, \mathscr{T}_{s}$ of pairwise orthogonal generalized standard stable tubes in $\Gamma_{C}$ such that $A$ is a generalized multicoil enlargement of $C$ using modules from $\mathscr{T}_{1}, \mathscr{T}_{2}, \ldots, \mathscr{T}_{s}$, and $\mathscr{C}$ is the generalized multicoil obtained from the stable tubes $\mathscr{T}_{1}, \mathscr{T}_{2}, \ldots, \mathscr{T}_{s}$ by an iterated application of the translation quiver admissible operations corresponding to the algebra admissible operations of types $(\operatorname{ad} 1)-(\operatorname{ad} 5)$ and $\left(\operatorname{ad} 1^{*}\right)-\left(\operatorname{ad} 5^{*}\right)$ leading from $C$ to $A$. From the definition of the generalized multicoil and [28, Lemmas 4.5, 4.6] we know that for the exceptional configuration of modules with the irreducible morphism $f$ we have the following mesh-complete full subquiver in $\mathscr{C}$

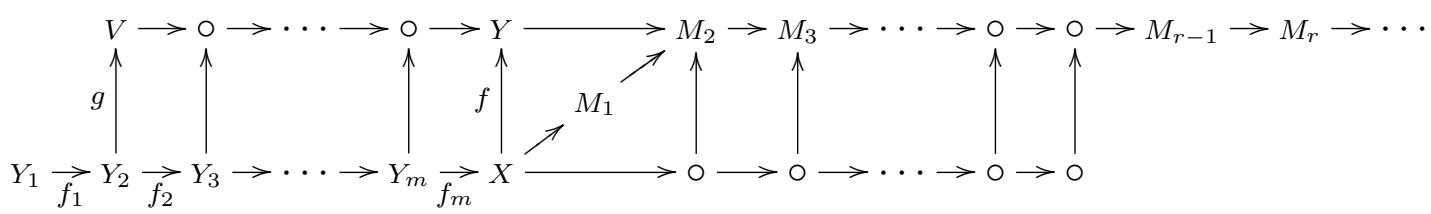

where $Y_{1} \stackrel{f_{1}}{\longrightarrow} Y_{2} \stackrel{f_{2}}{\longrightarrow} \cdots \stackrel{f_{m-1}}{\longrightarrow} Y_{m} \stackrel{f_{m}}{\longrightarrow} X$ is a sectional path of length $m$ such that $f f_{m} \ldots f_{1}=0$ for some integer $m \geq 1$, and $\alpha(g)=1$. Then, by Lemma 3.1, $d_{l}(f)=n$ if and only if $n=m$, getting the result.

Assume that $d_{r}(f)=n<\infty$. Then, there exists an indecomposable module $Z$ in $\mathscr{C}$ and a morphism $\varphi \in \Re^{n}(Y, Z) \backslash \Re^{n+1}(Y, Z)$ such that $\varphi f \in \Re^{n+2}(X, Z)$. Moreover, by [9, Proposition V. 7.4], there is a non-zero path of irreducible morphisms $\delta: Y \rightsquigarrow Z$ of length exactly $n$. Note that $f$ is an epimorphism. Indeed, applying Lemma 3.2 to the short exact sequences given by the meshes bounded by the sectional paths: $Y_{1} \rightarrow \cdots \rightarrow X, X \rightarrow Y$ and $V \rightarrow \cdots \rightarrow Y$ (see figure above) we get an exact sequence

$$
0 \longrightarrow Y_{1} \longrightarrow X \stackrel{f}{\longrightarrow} Y \longrightarrow 0
$$

Since $f$ is an irreducible epimorphism we also have that there is a non-zero path of irreducible morphisms of length $n+1$, given by the composition $X \stackrel{f}{\rightarrow} Y \stackrel{\delta}{\rightsquigarrow} Z$. Indeed, if $\delta f=0$ and $f$ is an epimorphism then $\delta=0$.

Now, since $\varphi f \in \Re^{n+2}(X, Z)$ and $f$ is an epimorphism then $\varphi f \neq 0$. Hence $\varphi f \in \Re^{n+k}(X, Z) \backslash \Re^{n+k+1}(X, Z)$, with $k \geq 2$, because $\mathscr{C}$ is generalized standard. Therefore, there exists a non-zero path of irreducible morphisms $X \rightsquigarrow Z$ of length exactly $n+k$, but any path from $X$ to $Z$ of length greater than $n+1$ in $\mathscr{C}$ is zero, which follows from the definition of the admissible operation (ad 5). More precisely, in order to obtain the considered exceptional configuration of modules we had to use an admissible operation (ad 5) in the following way: first we did one of the operations ( fad 2) or (fad 3) with the pivot $X$, next a finite number (possibly empty) of the operation (fad 4) and finally the operation (ad 4), and in such a way that the new projective vertices lie on a common infinite sectional path. From the definition of the operation (fad 2) (respectively, (fad 3)) we know that the set $\mathcal{S}_{\alpha}$ (respectively, $\mathcal{S}_{\beta}$ ) of all indecomposable modules $N$ such that there 
exists a non-zero homomorphism from $X$ to $N$ is of the form (see Section 2)

$$
\mathcal{S}_{\alpha}=\left\{X=X_{0}, X_{0}^{\prime}, X_{k}, X_{k}^{\prime}, Y_{l}, Y_{l}^{\prime}, Z_{k l} \mid 1 \leq k \leq s, 1 \leq l \leq t\right\},
$$

$s \geq 1, t \geq 1$ (respectively,

$$
\mathcal{S}_{\beta}=\left\{X=X_{0}, X_{0}^{\prime}, X_{m}, X_{m}^{\prime}, Y_{l}, Y_{l}^{\prime}, Z_{k l} \mid 1 \leq m \leq s, l \leq k \leq s, 1 \leq l \leq t\right\},
$$

$s \geq t-1, t \geq 2$ ). Therefore, after applying the admissible operation (ad 5) we get that the set $\mathcal{S}_{\alpha}^{\prime}$ (respectively, $\mathcal{S}_{\beta}^{\prime}$ ) of all indecomposable modules $M$ such that there exists a non-zero homomorphism from $X$ to $M$ in $\mathscr{C}$ consist the module $X_{0}^{\prime}$, the modules lying on the sectional path $X=X_{0} \rightarrow \cdots \rightarrow X_{s}, s \geq 1$, formed by arrows pointing to the mouth, the modules lying on the sectional path $X_{1}^{\prime} \rightarrow \cdots \rightarrow X_{s}^{\prime}$ formed by arrows pointing to infinity, and the modules lying on the infinite sectional paths $Y_{l} \rightarrow Z_{1 l} \rightarrow Z_{2 l} \rightarrow \cdots, 1 \leq l \leq t, t \geq 1$, formed by arrows pointing to infinity (respectively, consists the modules $X_{0}^{\prime}, \ldots, X_{t-1}^{\prime}, Y_{1}, \ldots, Y_{t}, t \geq 2$, the modules lying on the sectional path $X=X_{0} \rightarrow \cdots \rightarrow X_{s}, s \geq t-1$, formed by arrows pointing to the mouth, the modules lying on the sectional path $X_{t}^{\prime} \rightarrow \cdots \rightarrow X_{s}^{\prime}$ formed by arrows pointing to infinity, and the modules lying on the infinite sectional paths $Z_{l l} \rightarrow Z_{l+1, l} \rightarrow Z_{l+2, l} \rightarrow \cdots, 1 \leq l \leq t$, formed by arrows pointing to infinity). Hence, if there exists a non-zero path of irreducible morphisms from $X$ to $Z$, then $Z \in \mathcal{S}_{\alpha}^{\prime}$ or $Z \in \mathcal{S}_{\beta}^{\prime}$ and any longer path of irreducible morphisms from $X$ to $Z$ in $\mathscr{C}$ is zero. Thus, $d_{r}(f)=\infty$. This finishes the proof.

Lemma 3.4. Let $A$ be an algebra and $\mathscr{C}$ a generalized standard coherent almost cyclic component of $\Gamma_{A}$ with an exceptional configuration of modules of one of the forms

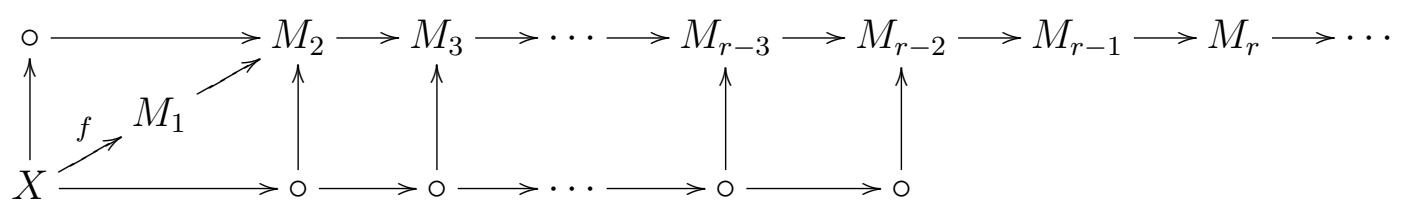

or

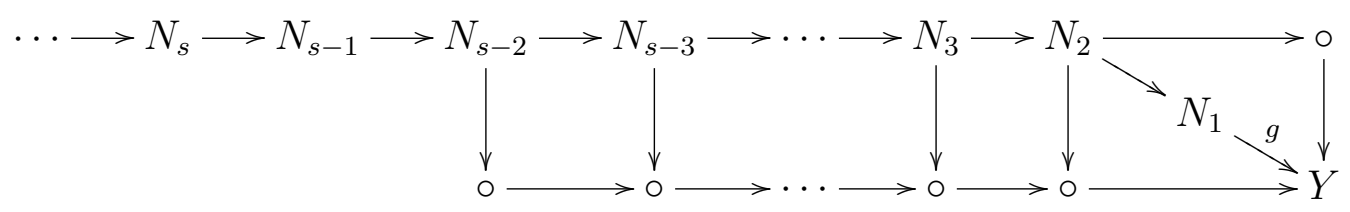

Then

(i) $d_{l}(f)=\infty$ and $d_{r}(f)=\infty$.

(ii) $d_{l}(g)=\infty$ and $d_{r}(g)=\infty$.

Proof. We shall prove only (i), because the proof of (ii) is dual. Let $\mathscr{C}$ be a generalized standard coherent almost cyclic component of $\Gamma_{A}$. As in the proof of the 
foregoing lemma, $\mathscr{C}$ is a faithful component of $\Gamma_{A}, A$ is a generalized multicoil enlargement of an algebra $C$ using modules from a family $\mathscr{T}_{1}, \mathscr{T}_{2}, \ldots, \mathscr{T}_{s}$ of pairwise orthogonal generalized standard stable tubes in $\Gamma_{C}$, and $\mathscr{C}$ is the generalized multicoil obtained from the stable tubes $\mathscr{T}_{1}, \mathscr{T}_{2}, \ldots, \mathscr{T}_{s}$ by an iterated application of the translation quiver admissible operations corresponding to the algebra admissible operations of types $(\operatorname{ad} 1)-(\operatorname{ad} 5)$ and $\left(\operatorname{ad} 1^{*}\right)-\left(\operatorname{ad} 5^{*}\right)$ leading from $C$ to $A$.

Since $M_{1}$ is projective we get that $d_{l}(f)=\infty$.

Assume $d_{r}(f)=n<\infty$. Then, there exists an indecomposable module $Z$ in $\mathscr{C}$ and a morphism $\varphi \in \Re^{n}\left(M_{1}, Z\right) \backslash \Re^{n+1}\left(M_{1}, Z\right)$ such that $\varphi f \in \Re^{n+2}(X, Z)$. Moreover, by [9, Proposition V. 7.4], there is a non-zero path of irreducible morphisms $\delta: M_{1} \rightsquigarrow Z$ of length exactly $n$. Note that from the definition of (ad 5) starting with $\left(\right.$ fad 2) (respectively, (fad 3)) we know that the set $\mathcal{S}_{\alpha}$ (respectively, $\mathcal{S}_{\beta}$ ) of all indecomposable modules $N$ such that there exists a non-zero homomorphism from $M_{1}$ to $N$ in $\mathscr{C}$ consist the modules lying on the following infinite sectional paths formed by arrows pointing to infinity (see Section 2): $M_{1}=X_{0}^{\prime} \rightarrow Z_{11} \rightarrow Z_{21} \rightarrow \cdots$, $Z_{1 l} \rightarrow Z_{2 l} \rightarrow \cdots$, for any $2 \leq l \leq t$, and $X_{1}^{\prime} \rightarrow X_{2}^{\prime} \rightarrow \cdots$ (respectively, $X_{l}^{\prime} \rightarrow Z_{l+1, l+1} \rightarrow Z_{l+2, l+1} \rightarrow Z_{l+3, l+1} \rightarrow \cdots$, for any $0 \leq l \leq t-1$, with $X_{0}^{\prime}=M_{1}$ and $X_{t}^{\prime} \rightarrow X_{t+1}^{\prime} \rightarrow \cdots$ ), where $X$ is the pivot of (fad 2) and $t \geq 1$ (respectively, (fad 3) and $t \geq 2$ ).

We have now two cases to consider. Assume first that $\varphi f \neq 0$. Since $\mathscr{C}$ is generalized standard, we get $\varphi f \in \Re^{n+k}(X, Z) \backslash \Re^{n+k+1}(X, Z)$, for some $k \geq 2$. Therefore, there exists a non-zero path of irreducible morphisms from $X$ to $Z$ of length exactly $n+k$, but any path from $X$ to $Z$ of length greater than $n+1$ in $\mathscr{C}$ is zero, as we have seen in the proof of the previous lemma. Assume now that there is a zero path of irreducible morphisms of length $n+1$, given by the composition $X \stackrel{f}{\rightarrow} M_{1} \stackrel{\delta}{\rightsquigarrow} Z$. Since the monomorphism $f: X \rightarrow M_{1}$ is fixed, it follows from the definition of (ad 5) with the considered exceptional configuration of modules that the path of length $n$ of irreducible morphisms from $M_{1}$ to $Z$ is zero. Then, any path of length $n$ in the generalized multicoil $\mathscr{C}$ from $M_{1}$ to $Z$ is zero, a contradiction. Therefore, we have $d_{r}(f)=\infty$. This finishes the proof.

\section{PROOF OF THEOREM 1.1}

Let $\mathscr{C}$ be a generalized standard coherent almost cyclic component of $\Gamma_{A}$. As in the proof of Lemma 3.3, we may assume that $\mathscr{C}$ is a faithful component of $\Gamma_{A}$. Then, by Theorem 2.3, there is an algebra $C$ (not necessarily indecomposable) and a family $\mathscr{T}_{1}, \mathscr{T}_{2}, \ldots, \mathscr{T}_{s}$ of pairwise orthogonal generalized standard stable tubes in $\Gamma_{C}$ such that $A$ is a generalized multicoil enlargement of $C$ using modules from $\mathscr{T}_{1}, \mathscr{T}_{2}, \ldots, \mathscr{T}_{s}$, and $\mathscr{C}$ is the generalized multicoil obtained from the stable tubes $\mathscr{T}_{1}, \mathscr{T}_{2}, \ldots, \mathscr{T}_{s}$ by an iterated application of the translation quiver admissible operations corresponding to the algebra admissible operations of types (ad 1)-(ad 5) and $\left(\operatorname{ad~} 1^{*}\right)-\left(\operatorname{ad} 5^{*}\right)$ leading from $C$ to $A$.

Let $f: X \rightarrow Y$ be an irreducible morphism in $\bmod A$ with $X, Y \in \mathscr{C}$. The proof will be carried out the same way as the proof of $[18$, Theorem A] with taking 
into account the possibility of appearing in $\mathscr{C}$ the exceptional configurations of modules. We have several cases to consider.

(a) Assume $\alpha(f)=1$ and $f$ is pointing to infinity. By [24, Proposition 1.12], we get $d_{l}(f)=1$. Let us calculate the right degree of $f$. It follows from our assumption on $\mathscr{C}$ that we have in this case one of the following three mesh-complete full subquivers in $\mathscr{C}$ for the irreducible morphism $f$

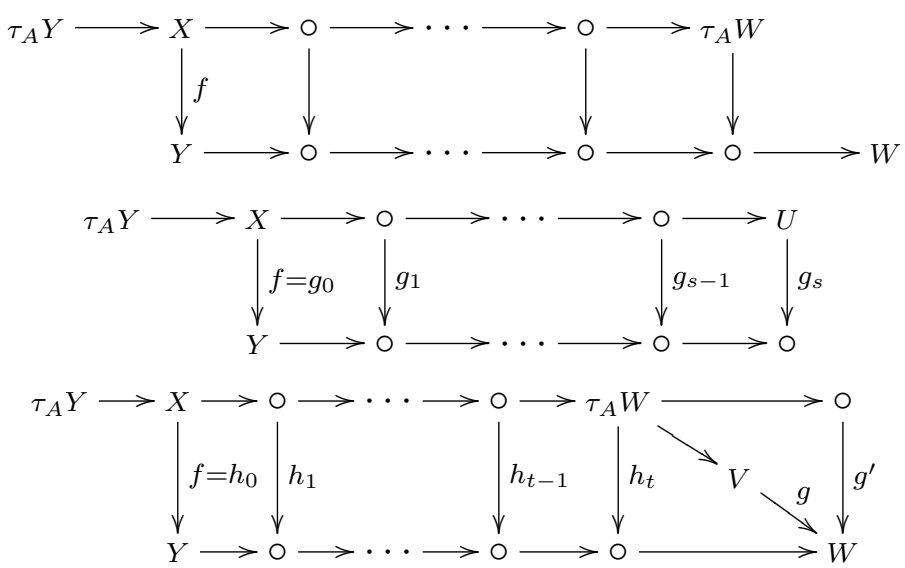

where $U$ is injective, and $s, t \geq 0$. It follows from the proof of [18, Theorem A] (the case (a)) that:

- the first configuration does not occur;

- for the second configurations we have $d_{r}(f)=\infty$;

- if $V$ is not injective, then for the third configurations we have $d_{r}(f)=\infty$.

Assume now that $V$ is injective. We claim that $d_{r}(f)=\infty$. First observe that $d_{r}\left(h_{t}\right)=\infty$. Indeed, if $d_{r}\left(h_{t}\right)<\infty$ then, by [24, Corollary 1.3], $d_{r}\left[\begin{array}{c}g^{\prime} \\ g\end{array}\right]<\infty$, and so $d_{r}\left(g^{\prime}\right)<\infty, d_{r}(g)<\infty$. But then we get a contradiction because, by the injectivity of $V, d_{r}(g)$ is infinite. Now, if $d_{r}(f)<\infty$ then, by the dual version of [24, Proposition 1.6],

$$
d_{r}(f)>d_{r}\left(h_{1}\right)>\ldots>d_{r}\left(h_{t-1}\right)>d_{r}\left(h_{t}\right)
$$

and we get a contradiction. Therefore, $d_{r}(f)=\infty$ and this proves our claim.

(b) Assume $\alpha(f)=1$ and $f$ is pointing only to the mouth. It follows from the proof of $\left[18\right.$, Theorem A] (the case (b)) that $d_{l}(f)=1$ and $d_{r}(f)=\infty$.

(c) Assume $\alpha(f)=2$ and $f$ is pointing to infinity. Let us calculate the left degree of $f$. It follows from our assumption on $\mathscr{C}$ and the proof of $[18$, Theorem A] (the case (c)) that we have in this case one more mesh-complete full subquiver in $\mathscr{C}$ for the irreducible morphism $f$, namely

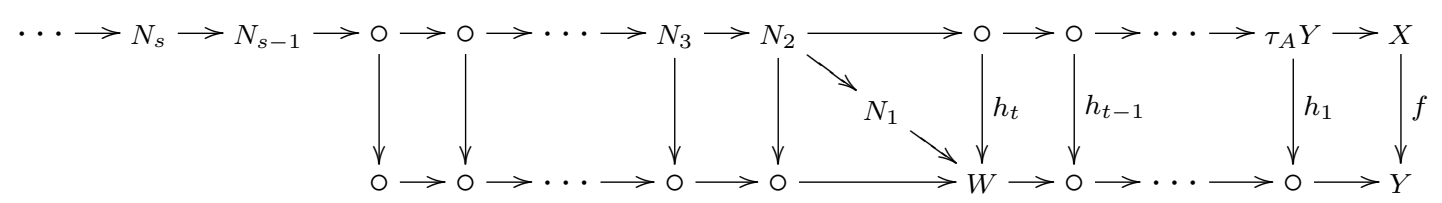


where $N_{1}$ and $N_{s}$ are injective, and $s \geq 4, t \geq 1$. We claim that $d_{l}(f)=\infty$. Indeed, it follows from Lemma 3.3(ii) that $d_{l}\left(h_{t}\right)=\infty$. Further, if $d_{l}(f)<\infty$ then, by [24, Proposition 1.6],

$$
d_{l}(f)>d_{l}\left(h_{1}\right)>\ldots>d_{l}\left(h_{t-1}\right)>d_{l}\left(h_{t}\right)
$$

and we get a contradiction. Hence, $d_{l}(f)=\infty$.

For the right degree of $f$ our claim follows from the proof of [18, Theorem A] (the case $(c)$ ).

(d) Assume $\alpha(f)=2$ and $f$ is pointing only to the mouth. Our claim follows from the proof of [18, Theorem A] (the case $(d)$ ).

(e) Assume $\alpha(f)=3$. If the irreducible morphism $f$ belongs neither to an exceptional configuration of modules nor to the mesh with exactly three middle terms created by an operation of type $(\operatorname{fad} 3)$ or $\left(\right.$ fad $\left.3^{*}\right)$, then our claim follows from the proof of $[18$, Theorem A] (the case (e)). Otherwise, we have to consider the following three subcases.

(e1) In the first subcase the component $\mathscr{C}$ contains the following mesh-complete full subquiver

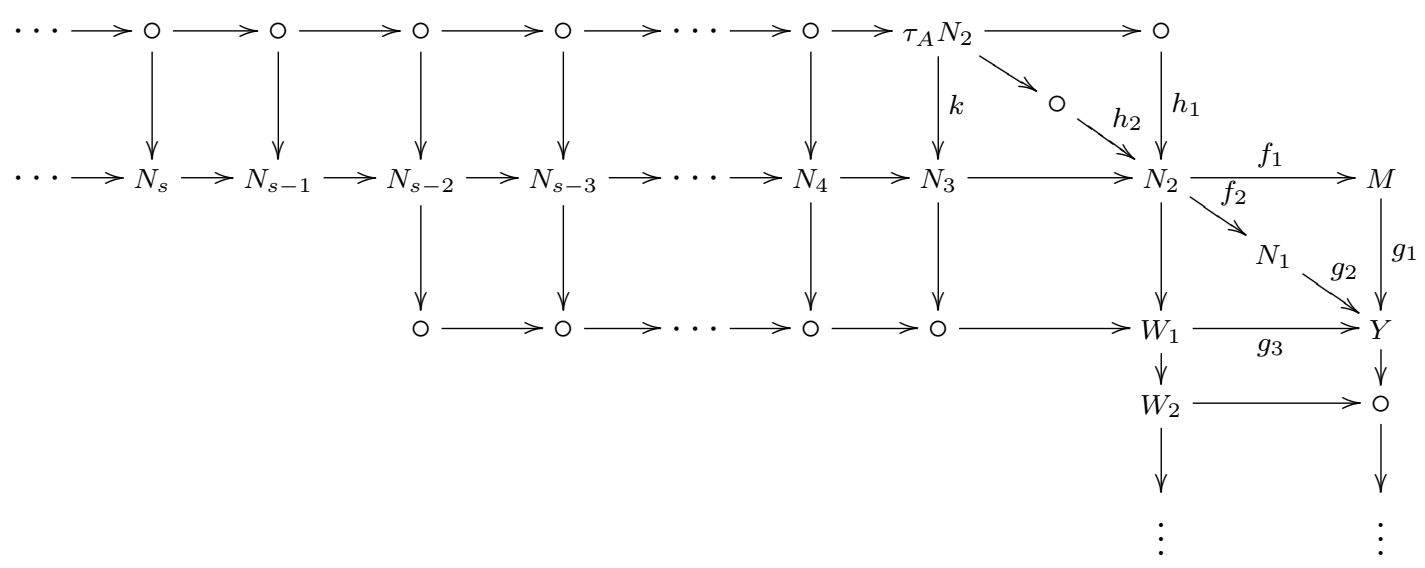

where $N_{1}, N_{s}$ are injective, $s \geq 4$, and $f \in\left\{g_{1}, g_{2}, g_{3}\right\}$.

Now, if $f=g_{1}, X=M$ then, by Lemma 3.3(ii) and its proof, we get $d_{l}\left(g_{1}\right)=\infty$ and $d_{r}\left(g_{1}\right)=n$ if and only if there is a sectional path

$$
Y=U_{0} \stackrel{u_{1}}{\longrightarrow} U_{1} \stackrel{u_{2}}{\longrightarrow} \cdots \stackrel{u_{n-1}}{\longrightarrow} U_{n-1} \stackrel{u_{n}}{\longrightarrow} U_{n}
$$

of irreducible morphisms in $\bmod A$ with $U_{1}, U_{2}, \ldots, U_{n}$ in $\mathscr{C}$ such that $u_{n} \ldots u_{1} g_{1}=$ 0 for some integer $n \geq 1$.

Further, if $f=g_{2}, X=N_{1}$ then, by Lemma 3.4(ii), we have $d_{l}\left(g_{2}\right)=\infty$ and $d_{r}\left(g_{2}\right)=\infty$.

Finally, we shall show that $d_{l}(f)=\infty$ and $d_{r}(f)=\infty$ for $f=g_{3}, X=W_{1}$. From the definition of a generalized multicoil we know that there exists the infinite sectional path $W_{1} \rightarrow W_{2} \rightarrow \cdots$ in $\mathscr{C}$ such that $Y \oplus W_{2}$ is a summand of $\epsilon^{\prime}\left(W_{1}\right)$. Then using the dual version of [24, Corollary 1.6] we receive that $d_{r}\left(g_{3}\right)=\infty$. We next prove that $d_{l}\left(g_{3}\right)$ is infinite. First observe that $d_{l}(k)$ is infinite. Indeed, by 
the definition of the exceptional configuration of modules there exists the infinite sectional path $\cdots \rightarrow N_{s} \rightarrow N_{s-1} \rightarrow \cdots \rightarrow N_{4} \rightarrow N_{3}$ in $\mathscr{C}$. Moreover, from the definition of a generalized multicoil we know that the above sectional path is such that $\tau_{A} N_{2} \oplus N_{4}$ is a summand of $\epsilon\left(N_{3}\right)$. Then using [24, Corollary 1.6] we get $d_{l}(k)=\infty$. Further, if $d_{l}\left(g_{3}\right)<\infty$ then, by [24, Corollary 1.2], $d_{l}\left[\begin{array}{l}f_{1} \\ f_{2}\end{array}\right]<\infty$ and, by [24, Lemma 1.11], $d_{l}\left[\begin{array}{l}h_{1} \\ h_{2}\end{array}\right]<\infty$. Hence, again by [24, Corollary 1.2], $d_{l}(k)<\infty$, a contradiction. Therefore, $d_{l}\left(g_{3}\right)=\infty$.

(e2) In the second subcase the component $\mathscr{C}$ contains the following meshcomplete full subquiver

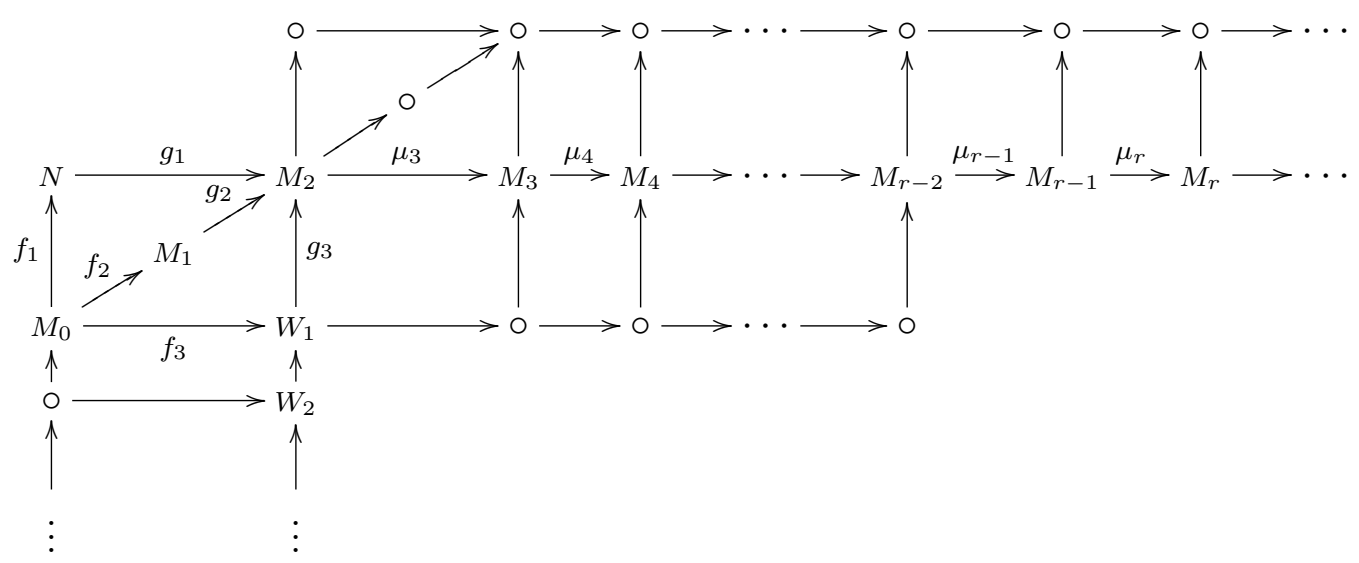

where $M_{1}, M_{r}$ are projective, $r \geq 4$, and $f \in\left\{g_{1}, g_{2}, g_{3}\right\}$.

Now, if $f=g_{1}, X=N, Y=M_{2}$ then, by [24, Proposition 1.12], we get $d_{r}\left(g_{1}\right)=1$. We claim that $d_{l}\left(g_{1}\right)$ is infinite. Assume $d_{l}\left(g_{1}\right)<\infty$. Then, by $[24$, Corollary 1.2], $d_{l}\left[\begin{array}{l}f_{2} \\ f_{3}\end{array}\right]<\infty$. Hence $d_{l}\left(f_{2}\right)<\infty$ and $d_{l}\left(f_{3}\right)<\infty$, but applying Lemma 3.4(i), we have $d_{l}\left(f_{2}\right)=\infty$. This contradiction proves our claim.

Further, if $f=g_{2}, X=M_{1}, Y=M_{2}$ then, again by [24, Proposition 1.12], we get $d_{r}\left(g_{2}\right)=1$. We next prove that $d_{l}\left(g_{2}\right)$ is infinite. First observe that $d_{l}\left(f_{3}\right)$ is infinite. Indeed, we know that in the sequence of admissible operations used to create the component $\mathscr{C}$, there is at least one operation of type $\left(\operatorname{ad} 1^{*}\right)$ or $\left(\operatorname{ad} 5^{*}\right)$ which contains the operation (fad $1^{*}$ ) which gives rise to the pivot $M_{0}$ of (fad 3 ) in the support of $\operatorname{Hom}_{A}\left(M_{0},-\right)$ restricted to the generalized multicoil containing $M_{0}$. The operations done after must not affect this support. Note that, in general, in the sequence of admissible operations used to create of $\mathscr{C}$ can be an operation of type (ad 5) which contains an operation ( $\mathrm{fad} 4$ ) which gives rise to the pivot $M_{0}$ of (fad 3) but from [29, Lemma 3.10] this case can be reduced to (ad $5^{*}$ ) which contains an operation (fad $\left.1^{*}\right)$. Therefore, in particular we have the infinite sectional path $\cdots \rightarrow W_{2} \rightarrow W_{1}$ in $\mathscr{C}$ such that $M_{0} \oplus W_{2}$ is a summand of $\epsilon\left(W_{1}\right)$. Then using $\left[24\right.$, Corollary 1.6] we get $d_{l}\left(f_{3}\right)=\infty$. Now, assume $d_{l}\left(g_{2}\right)<\infty$. Then, by $[24$, 
Corollary 1.2], $d_{l}\left[\begin{array}{l}f_{1} \\ f_{3}\end{array}\right]<\infty$. Hence $d_{l}\left(f_{1}\right)<\infty$ and $d_{l}\left(f_{3}\right)<\infty$, but $d_{l}\left(f_{3}\right)=\infty$ as shown above. This contradiction proves that $d_{l}\left(g_{2}\right)=\infty$.

Finally, if $f=g_{3}, X=W_{1}, Y=M_{2}$ then we have $\mu_{r-1} \ldots \mu_{4} \mu_{3} g_{3}=0$ for some integer $r \geq 4$, where

$$
M_{2} \stackrel{\mu_{3}}{\longrightarrow} M_{3} \stackrel{\mu_{4}}{\longrightarrow} \cdots \stackrel{\mu_{r-2}}{\longrightarrow} M_{r-2} \stackrel{\mu_{r-1}}{\longrightarrow} M_{r-1}
$$

is a sectional path of length $r-3$. By the dual version of Lemma 3.1, $d_{r}\left(g_{3}\right)=n$ if and only if $n=r-3$, getting the result. We claim that $d_{l}\left(g_{3}\right)=\infty$. Indeed, if $d_{l}\left(g_{3}\right)<\infty$ then, by [24, Corollary 1.2], $d_{l}\left[\begin{array}{l}f_{1} \\ f_{2}\end{array}\right]<\infty$. Hence $d_{l}\left(f_{1}\right)<\infty$ and $d_{l}\left(f_{2}\right)<\infty$, but applying Lemma 3.4(i), we have $d_{l}\left(f_{2}\right)=\infty$. This contradiction proves our claim.

(e3) In the last subcase the component $\mathscr{C}$ contains the following mesh-complete full subquiver

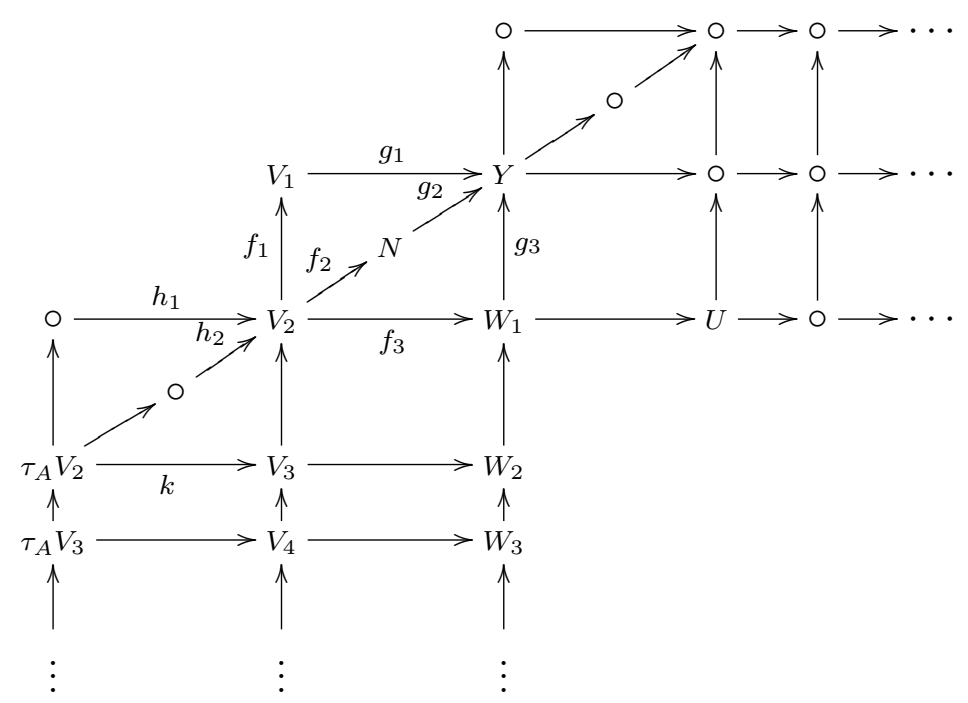

where $N$ is neither projective nor injective, and $f \in\left\{g_{1}, g_{2}, g_{3}\right\}$.

Now, if $f=g_{1}, X=V_{1}$ then, by [24, Proposition 1.12], we get $d_{r}\left(g_{1}\right)=1$. We shall show that $d_{l}\left(g_{1}\right)$ is infinite. First observe that $d_{l}\left(f_{3}\right)$ is infinite. Indeed, by the definition of a generalized multicoil we know that there exists the infinite sectional path $\cdots \rightarrow W_{2} \rightarrow W_{1}$ in $\mathscr{C}$ such that $V_{2} \oplus W_{2}$ is a summand of $\epsilon\left(W_{1}\right)$. Then using [24, Corollary 1.6] we get $d_{l}\left(f_{3}\right)=\infty$. Further, if $d_{l}\left(g_{1}\right)<\infty$ then, by [24, Corollary 1.2], $d_{l}\left[\begin{array}{l}f_{2} \\ f_{3}\end{array}\right]<\infty$. Hence $d_{l}\left(f_{2}\right)<\infty$ and $d_{l}\left(f_{3}\right)<\infty$, but $d_{l}\left(f_{3}\right)=\infty$ as shown above. This contradiction proves that $d_{l}\left(g_{1}\right)=\infty$.

If $f=g_{2}, X=N$ then, again by [24, Proposition 1.12], we get $d_{r}\left(g_{2}\right)=1$. We claim that $d_{l}\left(g_{2}\right)=\infty$. Indeed, if $d_{l}\left(g_{2}\right)<\infty$ then, by [24, Corollary 1.2], $d_{l}\left[\begin{array}{l}f_{1} \\ f_{3}\end{array}\right]<\infty$. Hence $d_{l}\left(f_{1}\right)<\infty$ and $d_{l}\left(f_{3}\right)<\infty$, but $d_{l}\left(f_{3}\right)=\infty$ as shown above. This contradiction proves our claim. 
Finally, we shall show that $d_{l}(f)=\infty$ and $d_{r}(f)=\infty$ for $f=g_{3}, X=W_{1}$. From the definition of a generalized multicoil we know that there exists the infinite sectional path $W_{1} \rightarrow U \rightarrow \cdots$ in $\mathscr{C}$ such that $Y \oplus U$ is a summand of $\epsilon^{\prime}\left(W_{1}\right)$. Then using the dual version of [24, Corollary 1.6] we receive that $d_{r}\left(g_{3}\right)=\infty$. We next prove that $d_{l}\left(g_{3}\right)$ is infinite. First observe that $d_{l}(k)$ is infinite. Indeed, again by the definition of a generalized multicoil we know that there exists the infinite sectional path $\cdots \rightarrow V_{4} \rightarrow V_{3}$ in $\mathscr{C}$ such that $\tau_{A} V_{2} \oplus V_{4}$ is a summand of $\epsilon\left(V_{3}\right)$. Then using [24, Corollary 1.6] we get $d_{l}(k)=\infty$. Further, if $d_{l}\left(g_{3}\right)<\infty$ then, by [24, Corollary 1.2], $d_{l}\left[\begin{array}{l}f_{1} \\ f_{2}\end{array}\right]<\infty$ and, by [24, Lemma 1.11], $d_{l}\left[\begin{array}{l}h_{1} \\ h_{2}\end{array}\right]<\infty$. Hence, again by [24, Corollary 1.2], $d_{l}(k)<\infty$, a contradiction. Therefore, $d_{l}\left(g_{3}\right)=\infty$.

We use dual arguments to compute all cases for $\alpha^{\prime}(f)$.

We note that in the course of the above proof the statements presented in Corollary 1.2 have been obtained.

\section{PROOF OF COROLLARY 1.3}

The proof is identical as in [18, Corollary C]. We shall repeat it here for the convenience of the reader. We shall prove only (i), because the proof of (ii) is dual.

If $d_{l}(f)=n$ then by Theorem 1.1 there is a sectional path

$$
Z_{0} \stackrel{g_{1}}{\longrightarrow} Z_{1} \stackrel{g_{2}}{\longrightarrow} \cdots \stackrel{g_{n-1}}{\longrightarrow} Z_{n-1} \stackrel{g_{n}}{\longrightarrow} Z_{n}=X
$$

of irreducible morphisms in $\bmod A$ with $Z_{0}, Z_{1}, \ldots, Z_{n-1}$ in $\mathscr{C}$ such that $f g_{n} \ldots g_{1}=$ 0 . Then $g_{n} \ldots g_{1}$ factors through Ker $f$. Thus, we have $g_{n} \ldots g_{1}=\iota \varphi$, where $\varphi: Z_{0} \rightarrow \operatorname{Ker} f$ and $\iota: \operatorname{Ker} f \rightarrow X$ is the inclusion morphism. Hence, we infer that $\iota \in \Re_{A}^{n}(\operatorname{Ker} f, X) \backslash \Re_{A}^{n+1}(\operatorname{Ker} f, X)$ and therefore $Z_{0} \cong \operatorname{Ker} f$.

If $\iota \in \Re_{A}^{n}(\operatorname{Ker} f, X) \backslash \Re_{A}^{n+1}(\operatorname{Ker} f, X)$ then $d_{l}(f) \leq n$. Assume that $d_{l}(f)=m<$ $n$. Again, by Theorem 1.1, there is a sectional path

$$
X_{0} \stackrel{h_{1}}{\longrightarrow} X_{1} \stackrel{h_{2}}{\longrightarrow} \cdots \stackrel{h_{m-1}}{\longrightarrow} X_{m-1} \stackrel{g_{m}}{\longrightarrow} X_{m}=X
$$

of irreducible morphisms in $\bmod A$ with $X_{0}, X_{1}, \ldots, X_{m-1}$ in $\mathscr{C}$ such that $f h_{m} \ldots h_{1}$ $=0$. Then $h_{m} \ldots g_{1}=\iota \psi$, where $\psi: X_{0} \rightarrow \operatorname{Ker} f$, a contradiction to the fact that $\iota \in \Re_{A}^{n}(\operatorname{Ker} f, X) \backslash \Re_{A}^{n+1}(\operatorname{Ker} f, X)$ proving the result.

\section{ACKNOWLEDGMENTS}

The first author gratefully acknowledge support from Universidad Nacional of Mar del Plata and CONICET. She is a resercher from CONICET.

The second author gratefully acknowledge support from the research grant DEC-2011/02/A/ST1/00216 of the Polish National Science Center. 


\section{REFERENCES}

[1] Assem, I., Simson, D., Skowroński, A. (2006). Elements of the Representation Theory of Associative Algebras 1: Techniques of Representation Theory, London Math. Soc. Student Texts Vol. 65. Cambridge: Cambridge Univ. Press.

[2] Assem, I., Skowroński, A. (1990). Minimal representation-infinite coil algebras. Manuscr. Math. 67: 305-331.

[3] Assem, I., Skowroński, A. (1993). Multicoil algebras, in: Representations of Algebras, Canad. Math. Soc. Conf. Proc., vol. 14, Amer. Math. Soc., Providence, RI, pp. $29-68$.

[4] Auslander, M. (1974). Representation theory of artin algebras II. Comm. Algebra 1: 269-310.

[5] Auslander, M., Reiten, I. (1975). Representation theory of Artin algebras III. Amost split sequences. Comm. Algebra 3: 239-294.

[6] Auslander, M., Reiten, I. (1977). Representation theory of Artin algebras IV. Invariants given by almost split sequences. Comm. Algebra 5: 443-518.

[7] Auslander, M., Reiten, I. (1977). Representation theory of Artin algebras V. Methods for computing almost split sequences and irreducible morphisms. Comm. Algebra 5: 519-554.

[8] Auslander, M., Reiten, I. (1978). Representation theory of Artin algebras VI. A functorial approach to almost split sequences. Comm. Algebra 6: 257-300.

[9] Auslander, M., Reiten, I., Smalø, S. O. (1995). Representation Theory of Artin Algebras. Cambridge Stud. Adv. Math. Vol. 36. Cambridge: Cambridge Univ. Press.

[10] Bautista, R. (1980). On irreducible maps. Bull. Amer. Math. Soc. (N. S.) 2: 177-180.

[11] Chaio, C. (2010). Degrees of irreducible morphisms in standard components. J. Pure Appl. Algebra 214: 1063-1075.

[12] Chaio, C. (2014). Degrees and composition of irreducible morphisms in almost presectional paths. Algebra Represent. Theory 17: 407-432.

[13] Chaio, C. (2015). A generalization of the composition of irreducible morphisms in regular components. Algebra Represent. Theory 18: 323-337.

[14] Chaio, C. (2015). Degrees in Auslander-Reiten components with almost split sequences of at most two middle terms. J. Algebra Appl. 14: 26p.

[15] Chaio, C., Coelho, F. U., Trepode, S. (2007). On the composite of two irreducible morphisms in radical cube. J. Algebra 312: 650-667.

[16] Chaio, C., Coelho, F. U., Trepode, S. (2008). On the composite of irreducible morphisms in almost sectional paths. J. Pure Appl. Algebra 212: 244-261.

[17] Chaio, C., Le Meur, P., Trepode, S. (2011). Degrees of irreducible morphisms and finite-representation type. J. London Math. Soc. (2) 84: 35-57.

[18] Chaio, C., Malicki, P., Skowroński, A. (2013). Degrees of irreducible morphisms in coherent almost cyclic Auslander-Reiten components. J. Algebra 391: 125-151.

[19] Chaio, C., Platzeck, M., Trepode, S. (2004). On the degree of irreducible morphisms. J. Algebra 281: 200-224.

[20] Chaio, C., Trepode, S. (2010). The composite of irreducible morphisms in standard components. J. Algebra 323: 1000-1011. 
[21] Coelho, F. U., Marcos, E. M., Merklen, H. A., Skowroński, A. (1994). Module categories with infinite radical square zero are of finite type. Comm. Algebra 22: 4511-4517.

[22] D'Este, G., Ringel, C. M. (1984). Coherent tubes. J. Algebra 87: 150-201.

[23] Igusa, K., Todorov, G. (1984). A characterization of finite Auslander-Reiten quivers. J. Algebra 89: 148-177.

[24] Liu, S. (1992). Degrees of irreducible maps and the shapes of the Auslander-Reiten quivers. J. London Math. Soc. 45: 32-54.

[25] Liu, S. (1993). Semi-stable components of an Auslander-Reiten quiver. J. London Math. Soc. 47: 405-416.

[26] Malicki, P. (1998). Generalized coil enlargements of algebras. Colloq. Math. 76: 5783.

[27] Malicki, P. (2006). On the composition factors of indecomposable modules in almost cyclic coherent Auslander-Reiten components. J. Pure Appl. Algebra 207: 469-490.

[28] Malicki, P., Skowroński, A. (2000). Almost cyclic coherent components of an Auslander-Reiten quiver. J. Algebra 229: 695-749.

[29] Malicki, P., Skowroński, A. (2005). Algebras with separating almost cyclic coherent Auslander-Reiten components. J. Algebra 291: 208-237.

[30] Malicki, P., Skowroński, A. (2007). On the additive categories of generalized standard almost cyclic coherent Auslander-Reiten components. J. Algebra 316: 133-146.

[31] Malicki, P., Skowroński, A. (2007). Concealed generalized canonical algebras and standard stable tubes. J. Math. Soc. Japan 59: 521-539.

[32] Malicki, P., Skowroński, A. (2011). On the indecomposable modules in almost cyclic coherent Auslander-Reiten components, J. Math. Soc. Japan 63: 1121-1154.

[33] Malicki, P., Skowroński, A. (2011). Algebras with separating Auslander-Reiten components, in: Representations of Algebras and Related Topics, European Math. Soc. Series Congress Reports, European Math. Soc. Publ. House, Zürich, pp. 251-353.

[34] Ringel, C. M. (1984). Tame Algebras and Integral Quadratic Forms, Lecture Notes in Math., Vol. 1099, Springer-Verlag, Berlin-Heidelberg.

[35] Ringel, C. M. (1990). The canonical algebras, with an appendix by W. CrawleyBoevey in: Topics in Algebra, Part 1: Rings and Representations of Algebras, Banach Center Publ. Vol. 26, PWN, Warsaw, pp. 407-432.

[36] Skowroński, A. (1994). Generalized standard Auslander-Reiten components. $J$. Math. Soc. Japan 46: 517-543.

[37] Skowroński, A. (1994). On the composition factors of periodic modules. J. London Math. Soc. (2), 49: 477-492.

[38] Skowroński, A. (2001). Generalized canonical algebras and standard stable tubes. Colloq. Math. 90: 77-93.

[39] Skowroński, A., Zwara, G. (2000). Degeneration-like orders on the additive categories of generalized standard Auslander-Reiten components. Arch. Math. (Basel) 74: $11-21$. 